\title{
Liquid Crystal Materials in THz Technologies
}

\author{
Urszula Chodorow, ${ }^{* 1}$ Janusz Parka, ${ }^{1,2}$ and Olga Chojnowska ${ }^{3}$ \\ ${ }^{1}$ Institute of Applied Physics, Military University of Technology, Kaliskiego 2, 00-908 Warszawa, \\ ${ }^{2}$ Institute of Microelectronics and Optoelectronics, Warsaw University of Technology, Nowowiejska 12, \\ 00-667 Warszawa \\ ${ }^{3}$ Institute of Chemistry, Military University of Technology, Kaliskiego 2, 00-908 Warszawa,
}

Received August 31, 2012; accepted September 21, 2012; published September 30, 2012

\begin{abstract}
In this paper the results of measurements of three liquid crystal mixtures at the $\mathrm{THz}$ frequency range are presented. The influence of various components for optical properties like refractive indices and absorption from 0.3 to $3.0 \mathrm{THz}$ of nematic liquid crystal mixtures are shown. Chosen applications of liquid crystals in the $\mathrm{THz}$ range have been described.
\end{abstract}

The interest of terahertz $(\mathrm{THz})$ radiation has increased in the last decade. The $\mathrm{THz}$ region is between a microwave and an infrared frequency range and it is the least explored spectrum region. $\mathrm{THz}$ technology is now developing rapidly, there is high requirement for devices which modulate and control the $\mathrm{THz}$ frequency region. Potential applications of the $\mathrm{THz}$ range enclose: detection of weapons and illegal chemical and biological substances, medical imaging, epithelium cancer screening, productive processes control, etc. These applications require different active and passive optic elements such as lenses, phase shifters, modulators, filters, phase gratings, switches, etc. A good solution seems to utilize liquid crystals because of their high dielectric anisotropy and possibility of tuning by an electric or magnetic field $[1-4]$.

The measurements of liquid crystals (LCs) properties were performed using a conventional Time-Domain Spectrometer (TDS) at room temperature. The setup was described in many books and papers [5-7]. The LC cells for these measurements consisted of two quartz plates, each $1.5 \mathrm{~mm}$ thick. The thickness of a liquid crystal layer which was sandwiched between two quartz plates was $0.5 \mathrm{~mm}$. Copper wires as separators and electrodes were used. Homogeneous alignment was obtained by using a high voltage of about $30 \mathrm{kV} / \mathrm{m}$. The reference used was two directly- adjacent quartz plates, each of $1.5 \mathrm{~mm}$ thickness.

The result of measurement is an electro-optical signal versus time (Fig. 1). By using Fast Fourier Transform (FFT), amplitude (real part of FFT) and phase (imaginary part of FFT) are respectively obtained versus frequency.

*E-mail: uchodorow@wat.edu.pl
From these data, parameters like refractive indices $n_{L C}$ and absorption coefficients $\alpha_{L C}$ are calculated by using Eqs. (1) and (2):

$$
\begin{gathered}
n_{L C}=1+\frac{c}{\omega d}\left(\varphi_{\text {samp }}-\varphi_{r e f}\right), \\
\alpha_{L C}=-\frac{2}{d} \ln \left(\frac{\left(n_{q}+n_{L C}\right)^{2}}{4 n_{q} n_{L C}} \cdot\left|\frac{E_{\text {samp }}}{E_{r e f}}\right|\right),
\end{gathered}
$$

where $c$ is the light speed in vacuum, $d$ is the thickness of $\mathrm{LC}$ layer, $\omega$ is the angular frequency of $\mathrm{THz}$ wave, $\varphi_{\text {samp}}$, $\varphi_{\text {ref }}$ and $E_{\text {samp }}, E_{\text {ref }}$ are the phases and amplitudes of the $\mathrm{THz}$ field passing through the sample and reference respectively, and $n_{q}$ is the refractive index of the quartz plate.

The coefficients for ordinary and extraordinary rays were obtained by using the same Eqs. (1) and (2). The ordinary coefficients were measured when the polarization of a $\mathrm{THz}$ wave was perpendicular to an LC director. When the polarization of an incident $\mathrm{THz}$ wave was parallel to an LC director, extraordinary indices were obtained.

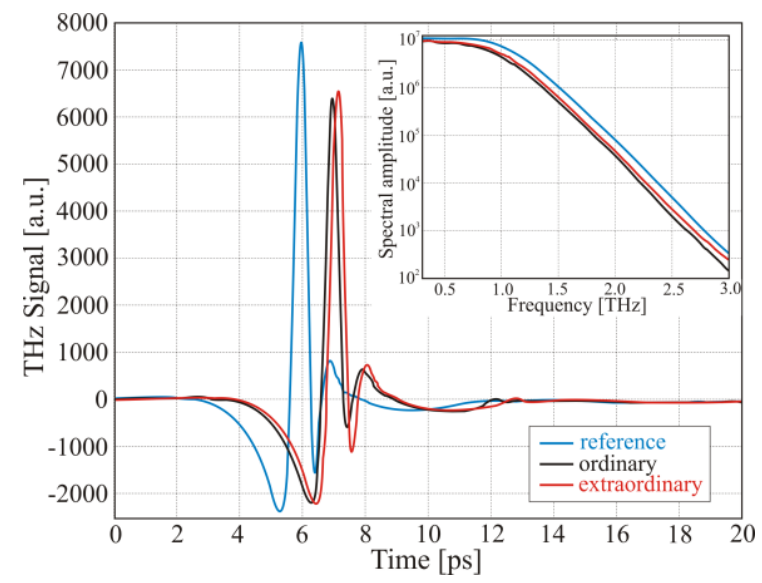

Fig. 1. THz waveform transmitted through reference and sample cell with LC mixture 1855 . 
The composition of LC mixtures is presented in Fig. 2. Liquid crystal mixture 1855 consists of three molecules with a different alkyl chain length for $n=5, n=3$ and $n=2$. The details of the 1855 can be found in Ref. [8]. Liquid crystal mixtures $1855 \mathrm{~A}$ and $1855 \mathrm{D}$ consist of 1855 and molecules presented in Fig. $2 \mathrm{~b}$ and Fig. 2c, respectively. The measured materials were synthesized in the Military University of Technology in Warsaw by R. Dabrowski's team.

a)

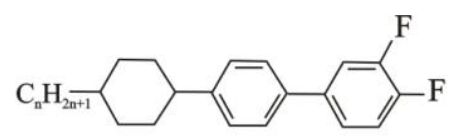

b)

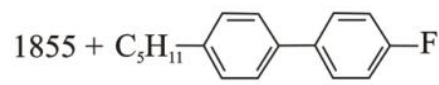

c)

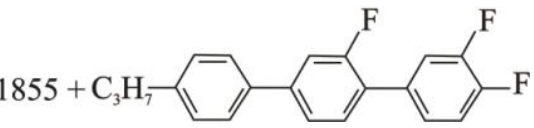

Fig. 2. Composition of LC mixtures a) 1855 (for $n=5,3$ and 2), b) $1855 \mathrm{~A}$ and c) $1855 \mathrm{D}$.

In Fig. 3 refractive indices versus frequency for ordinary $n_{o}$ and extraordinary $n_{e}$ ray for measured LC mixtures are presented. The coefficients for an ordinary ray decrease with the increasing frequency for all LC samples. Extraordinary indices have a tendency to increase with the increasing frequency. Refractive indices for an ordinary ray have similar values for 1855 and $1855 \mathrm{~A}$ over the $0.3-3.0 \mathrm{THz}$ range. For $1855 \mathrm{D}$ the value of $n_{o}$ is slightly smaller. LC mixture 1855 has the highest values of $\mathrm{n}_{\mathrm{e}}$. The admixture molecules without lateral bonds caused the decreasing of $n_{e}$ in the case of 1855A. The smallest value of $n_{e}$ has $1855 \mathrm{D}$ with an admixture of molecules with two fluorine atoms linked in lateral positions to two phenyl rings.

In Fig. 4 , the values of birefringence $\Delta n$ versus frequency for all the LC samples are shown. LC mixtures 1855 and 1855D have the highest and smallest values of $\Delta n$ respectively. For $1855 \mathrm{D}$, a faster increase of $\Delta n$ with an increasing frequency is observed than in the case of 1855 and $1855 \mathrm{~A}$. This may be due to the occurrence of two additional lateral bonds of fluorine atoms in the additional molecules in the case of $1855 \mathrm{D}$. The slowest increase of birefringence is observed for 1855A. In this case, additional molecules have no lateral bonds. Therefore, it is possible that admixture molecules with and without lateral bonds of fluorine atoms influence the speed of $\Delta n$ change with the change of frequency.

Absorption coefficients versus frequency for ordinary $\alpha_{o}$ and extraordinary $\alpha_{e}$ ray for measured LC mixtures are presented in Fig. 5. For all LC mixtures $\alpha_{o}$ is always larger than $\alpha_{e}$. In the case of 1855A, decreased absorption for both ordinary and extraordinary ray as compared with
1855 is observed. $1855 \mathrm{D}$ has bigger absorption for an ordinary ray than the other LC mixtures and lower $\alpha_{e}$ than 1855. Therefore, the admixture of molecules with lateral bonds of fluorine atoms caused a large increase of absorption for an ordinary ray. The additional molecules without lateral bonds caused decreased absorption for both ordinary and extraordinary rays.

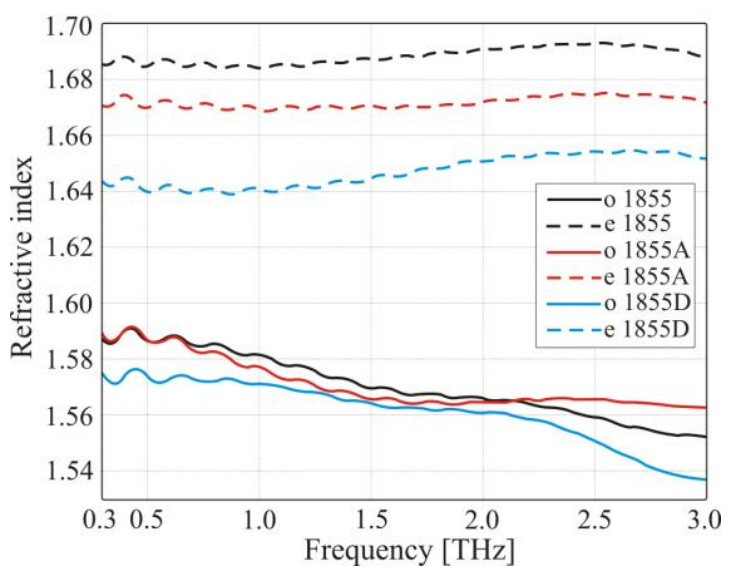

Fig. 3. Refractive indices for 1855, 1855A and 1855D (o-ordinary, e-extraordinary).

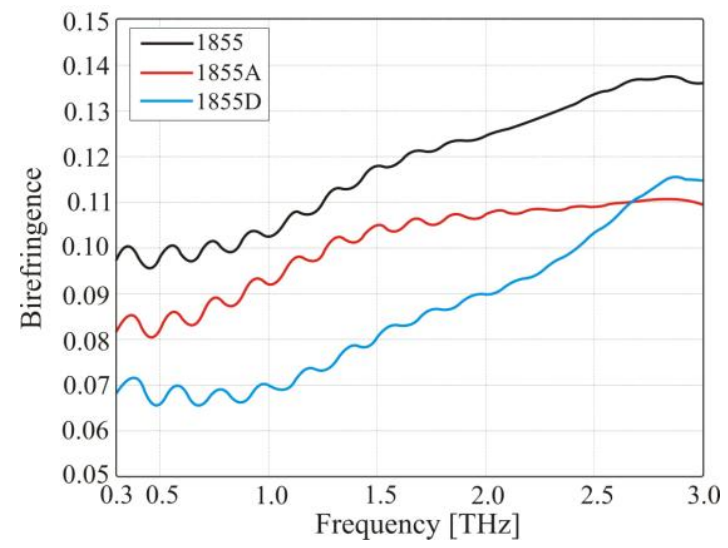

Fig. 4. Birefringence for 1855, 1855A and 1855D.

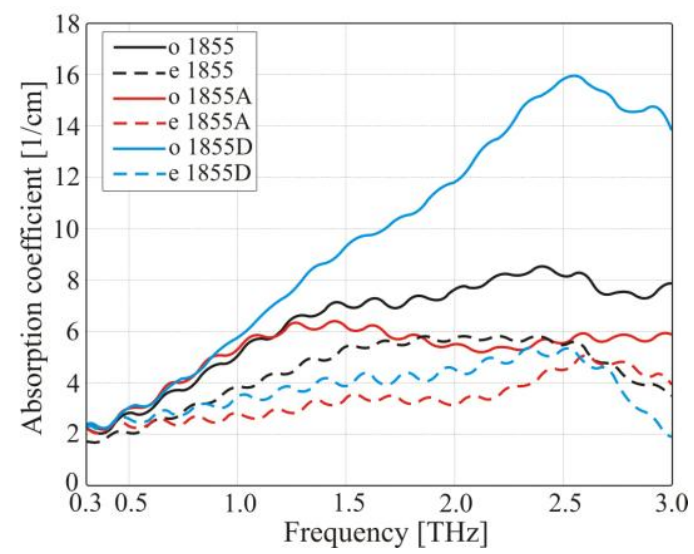

Fig. 5. Absorption coefficients for 1855, 1855A, 1855D (o-ordinary, e-extraordinary). 
In the literature there is a lot of solutions for utilizing LCs in devices in the $\mathrm{THz}$ region. In this paper two of them were described.

The first is a tunable filter for the $\mathrm{THz}$ range which uses a dual-frequency switching liquid crystal (DFSW-LC) [9]. Dual-frequency LCs have a negative or positive value of dielectric anisotropy $\Delta \varepsilon$ which depends on frequency steering voltage. For small frequencies (in this case $1 \mathrm{kHz}$ ) LCs have positive $\Delta \varepsilon$, and the long axes of molecules are organized parallel to the vector of the applied electric field. For high frequencies (in this case $40 \mathrm{kHz}$ ) LCs have negative $\Delta \varepsilon$, and LC director is perpendicular to the vector of the applied electric field. Using two voltage frequencies to control the anisotropy of LC allows much faster changes of LC orientation than other techniques, because the change of LC orientation is always forced by the electric field and not by the spontaneous reorganization of the molecules. Such a solution is advantageous because it eliminates the need for two external voltage sources, which facilitates the implementation of the measuring system.

The described filter is based on a Fabry-Perot effect in the gap formed by two metallic gold comb structures at the top and bottom of the two quartz plates. The comb teeth are oriented parallel to the electric field of the incident $\mathrm{THz}$ wave, and they act as a reflective mirror for $\mathrm{THz}$ signal. The thickness of the liquid crystal layer is $100 \mu \mathrm{m}$. The bandwidth filter for homeotropic and homogeneous orientation of $\mathrm{LC}$ is around $0.450 \mathrm{THz}$ and $0.466 \mathrm{THz}$, respectively. The tuning range is $16 \mathrm{GHz}$ with LC birefringence $\Delta n=0.08$. For homeotropic LC orientation, the dielectric losses are much higher, approximately twice as large as in the case of a homogeneous orientation of LC. Improving the parameters of liquid crystals, reducing absorption and increasing the anisotropy of electric permittivity will increase the scope of retuning this type of equipment and increase their usefulness in terahertz applications.

The other solution is a magnetically tunable $2 \pi$ LC phase shifter [10]. The LC phase shifter consists of two homeotropic LC (E7) cells each of a thickness of $1.5 \mathrm{~mm}$ and a rotating magnet. The applied magnetic field in the middle of LC was $0.427 \mathrm{~T}$ (threshold magnetic field equal to about $0.01 \mathrm{~T})$. The maximum possible angle of the rotation of the magnet was $\theta=54^{\circ}$ (the angle of inclination of magnetic field to the direction of $\mathrm{THz}$ wave propagation). Phase shift $\delta(\theta)$ depends on LC thickness, refractive indices changes and incident wave frequency. For the above parameters of the device phase shift was $\delta(\theta)=368^{\circ}$ for $1.025 \mathrm{THz}$ for maximum inclination angle of the magnetic field.
In this paper results of three LC mixtures measurements at the $\mathrm{THz}$ range have been presented. A great advantage of measured LC mixtures is low absorption, on the other hand, they also have low anisotropy of refractive indices below $\Delta n=0.14$. When the birefringence is small, the thicker LC layer is required in possible applications such as phase shifters.

The development of terahertz technology requires the construction of different devices which modulate and control the $\mathrm{THz}$ beam. In the last decade several tunable devices based on liquid crystals have been created. In this work two of them were described. But still, these systems are slow due to the use of thick layers of liquid crystals. The devices require high control voltages and have high losses. The control of LC by a magnetic field becomes difficult because of using more complex systems than those described in this paper.

We suppose that it is possible to synthesize liquid crystal materials which will have larger dielectric anisotropy and lower absorption in the $\mathrm{THz}$ range than those described above. It will enable to diminish the thickness of the applied LC layer, which will decrease control voltage and increase the speed of LC tunability.

The authors thank N. Palka for providing a $\mathrm{THz}$ spectrometer and valuable guidance. This article was financially supported by the Polish Ministry of Sciences and Higher Education, No. O R00 014412 "Tunable liquid crystal transducers for $\mathrm{THz}$ and $\mathrm{GHz}$ frequency range" and the Key Project POIG.01.03.01-14-016/08 "New Photonic Materials and their Advanced Applications".

\section{References}

[1] C.-Y. Chen, C.-L. Pan, C.-F. Hsieh, Y.-F. Lin, R.-P. Pan, Appl. Phys. Lett. 88, 101107 (2006).

[2] C.-J. Lin, C.-H. Lin, Y.-T. Li, R.-P. Pan, C.-L. Pan, IEEE Photon Technol. Lett. 21, 730 (2009).

[3] R. Wilk, N. Vieweg, O.Kopschinski, M. Koch, Opt. Exp. 17, 9 (2009).

[4] H. Li, Ch. Zhu, K. Liu, X. Zhang, F. Ling, T. Zhang, X. Shen, C. Zhang, S. Ruan, Infrared Phys. Techn. 54, 439 (2011).

[5] Y.-Sh. Lee, Principles of Terahertz Science and Technology (Oregon, Springer Science 2009).

[6] U. Chodorow, J. Parka, K. Garbat, N Pałka, K. Czupryński, L. Jaroszewicz, Mol. Cryst. Liq. Cryst. 561, 1 (2012).

[7] N. Pałka, T. Trzcinski, Proc. SPIE 79380, 79380L (2011).

[8] O. Chojnowska, R. Dąbrowski, Phot. Lett. Poland 4, 2 (2012).

[9] T. Gobel, P. Meissner, A. Gaebler, M. Koeberle, S. Muller, R. Jakoby, CLEO/QELS, 5224708 (2009).

[10] C.-Y. Chen, C.-F. Hsieh, Y.-F. Lin, R.-P. Pan, C.-L. Pan, Opt. Exp. 12, 12 (2004) 